\title{
Low Serum Cholesterol Concentration in Adult Patients with Phenylketonuria- One Centre Experience
}

\author{
Karolina M Stepien ${ }^{1 *}$ and Chris J Hendriksz ${ }^{1,2}$ \\ ${ }^{1}$ Adult Inherited Metabolic Disorders, The Mark Holland Metabolic Unit, Salford Royal Foundation NHS Trust, Ladywell NW2, Salford, Manchester, UK \\ ${ }^{2}$ Paediatrics and Child Health, University of Pretoria, Steve Biko Academic Unit, Pretoria, South Africa
}

\begin{abstract}
Introduction: Phenyloketonuria is a rare metabolic disorder caused by a deficiency of the phenylalanine (Phe) hydroxylase enzyme. A reduced intake of natural proteins helps optimise plasma Phe concentration. A relationship between high plasma Phe level and the inhibition of cholesterol synthesis was previously observed but the mechanisms are unclear. Low LDL-cholesterol concentrations were observed in children and adolescent PKU patients, but not in adults. This is the first paper to present lipid profile in adult patients with this condition.
\end{abstract}

Methods: Lipid profile was analysed in adult patients with PKU. We examined associations between Phe and four outcomes: total cholesterol, LDL-cholesterol, HDL-cholesterol and triglycerides. Confounding factors (predictors) were taken into account: body mass index (BMI), age and gender. The statistical analysis was performed using multiple linear regression.

Results: Among 176 adult patients 91 were females (52\%). The mean age was $32 \pm 10.7$ years. 82 patients (46\%) were on strict PKU diet. Mean Phe was $1017 \pm 440 \mu \mathrm{mol} / \mathrm{L}$. Mean total cholesterol was $4.33 \pm 0.94 \mathrm{mmol} / \mathrm{L}$, LDL-cholesterol $2.48 \pm 0.8 \mathrm{mmol} / \mathrm{L}$, HDL-cholesterol $1.2 \pm 0.34 \mathrm{mmol} / \mathrm{L}$, triglycerides $1.6 \pm 0.9 \mathrm{mmol} / \mathrm{L}$. There was no correlation between Phe concentration and lipid profile in our cohort of adult patients with PKU. No cardiovascular events were documented in our cohort.

Conclusions: In conclusion, the outcomes of the study demonstrate that mean total cholesterol, LDL-cholesterol and triglycerides concentrations were substantially lower as compared to healthy population, which may confer their reduced cardiovascular risk. Lipid profile remained low irrespectively of Phe concentration.

Keywords: PKU; Phenyloketonuria; Cardiovascular risk; Cholesterol; Lipids; BMI

Abbreviations: PKU: Phenyloketonuria; Phe: Phenylalanine; BMI: Body Mass Index; LDL-cholesterol: Low density lipoprotein cholesterol; HDL-Cholesterol: High density lipoprotein cholesterol.

\section{Introduction}

Phenyloketonuria (OMIM 261600) is a rare autosomal recessive metabolic disorder caused by a deficiency of the phenylalanine (Phe) hydroxylase enzyme (EC 1.14.16.1). Treatment consists of a reduced intake of natural proteins [1]. Animal protein is replaced by amino-acid formulas that consist of Phe-free amino acid mixtures supplemented with tyrosine, vitamins and other micronutrients [1]. As a result of reduced animal lipids consumption, plasma cholesterol concentration is low [2,3]. A relationship between high plasma Phe level and the inhibition of cholesterol synthesis has been previously described [47]. It has been hypothesized that two main regulatory enzymes in the cholesterol synthesis, i.e., 3-hydroxy-3-methylglutaryl-CoA-reductase and mevalonate-5-pyrophosphate decarboxylase in brain and liver, are suppressed by high plasma Phe $[4,6]$.

An inverse association between raised Phe and plasma cholesterol has been reported previously in children with PKU [2,3,8-12]. In adult patients, however, low-normal cholesterol concentration with no correlation between Phe and plasma cholesterol levels has been observed [13]. The controversy exists as to whether the hypocholesterolaemia is due to a disruption to cholesterol biosynthesis or the low protein diet. Most studies attribute low cholesterol levels in treated PKU patients to their vegetarian diet $[8,10]$. However, serum cholesterol concentrations depend on other regulatory factors such as the cholesterol synthesis, comorbidities such as diabetes or familial hyperlipidaemia, obesity, alcohol consumption. Obesity, which leads to metabolic syndrome, has been previously shown to be a common finding among children and adolescents with PKU $[14,15]$ irrespective of their compliance with low-protein diet [15]. Adolescent patients with PKU have been shown to have significantly low LDL-cholesterol compared to healthy controls $[15,16]$. HDL-cholesterol concentration that confers a low cardioprotective risk has not been different from healthy controls [15] or has been low in young patients compliant to low-protein diet compared with healthy controls and non-compliant patients [16]. In addition, raised homocysteine concentration and platelets counts were high in adolescent PKU patients that might increase the risk of atherosclerosis in this population [16]. However, Htun et al. have demonstrated that, despite low HDL-cholesterol concentrations and high triglycerides concentrations, adolescents with well-controlled PKU have normal carotid intima thickness and B-stiffness index [17]. In addition, platelet activation has not been enhanced meaning early atherosclerotic changes have not been induced [17]. In the present retrospective case notes review, we have explored the relationship between plasma Phe, diet, body mass index (BMI), age, gender and cholesterol in adult patients with PKU attending our Metabolic Clinic.

*Corresponding author: Karolina M Stepien, Adult Inherited Metabolic Disorders, The Mark Holland Metabolic Unit, Salford Royal Foundation NHS Trust, Ladywell NW2, 2nd Floor, Room 112, Salford, Manchester, UK, Tel: 01612064365; Fax: 01612064036; E-mail: kstepien@doctors.org.uk

Received June 29, 2017; Accepted July 10, 2017; Published July 14, 2017

Citation: Stepien KM, Hendriksz CJ (2017) Low Serum Cholesterol Concentration in Patients with Phenylketonuria- One Centre Experience. Clin Med Biochem 3: 130. doi:10.4172/2471-2663.1000130

Copyright: (c) 2017 Stepien KM et al. This is an open-access article distributed under the terms of the Creative Commons Attribution License, which permits unrestricted use, distribution, and reproduction in any medium, provided the original author and source are credited. 


\section{Methods}

\section{Study design}

It was a retrospective observational study and a review of our clinical practice. All patients have their blood tests (lipid profile) requested as part of their routine care when attend our Metabolic Clinics appointments every 6 months. We follow our local protocol that was implemented as our clinical guidelines after input from the paediatric metabolic team who previously cared for the majority of these patients.

\section{Patients}

Demographic data for 176 adult PKU patients were extracted from the Electronic Patient Record and included in the analysis. Data included: BMI $\left(\mathrm{kg} / \mathrm{m}^{2}\right)$, age, gender and adherence to PKU diet. Pregnant PKU patients were excluded from the study.

\section{Biochemical tests}

Lipid profile included total cholesterol, LDL-cholesterol, HDLcholesterol and triglycerides were analysed using enzymatic method on Siemens Advia 2400 automated analyser in Clinical Biochemistry Department. Plasma Phe samples was collected on dry blood spot cards and was detected by multiple reaction monitoring mode, on a Waters Acquity-TQD tandem mass spectrometer in the Willink Biochemical Genetics Laboratory.

\section{Statistical analysis}

The statistical analysis was performed using multiple linear regression (Stats Direct 3 Statistical Software). We fitted four models, with each of total cholesterol, LDL-cholesterol, HDL-cholesterol and triglycerides as outcome variables. Phe was centred by subtracting the mean value, and was entered as a continuous predictor variable. We included interactions with Phe $\mathrm{x}$ gender, to allow for the possibility that the relationship between Phe might vary according to this variable. We additionally controlled for age and BMI as potential confounding variables. The variables to be included were selected on a priori grounds to answer the research question of the present study. We did not perform any automated variable selection, as this is known to be a poor procedure that produces spurious (due to multiple testing) and nongeneralizable (due to overfitting) results [18]. Results were presented as regression coefficients and 95\% CIs, which give ranges of values for the effects that are consistent with the study data.

\section{Results}

\section{Baseline characteristics}

Patients: Among 176 patients 91 were females (52\%). All patients were adult ( $>16$ years) and the mean age was $32 \pm 10.7$ years (range 17-64 years). 82 patients (46\%) were on strict PKU diet; no meat and no fish (Table 1). Patients often on vegetarian diet and had 2-3 amino acid supplements per day. None of our patients had documented cardiovascular disease such as myocardial infarction, stroke or angina.

Biochemical tests: Lipid profile and Phe results were expressed as mean $( \pm S D)$ and are summarized in (Table 1). Mean total cholesterol was comparable with the target $(<5 \mathrm{mmol} / \mathrm{L})$ determined for nonmetabolic patients [13]. Mean LDL-cholesterol was within the target ( $<3 \mathrm{mmol} / \mathrm{L}$ ) for healthy non-metabolic population.

Estimated associations: There was no evidence of an association between plasma Phe and four fractions of lipids profile after confounding factors such as BMI, age and gender were taken into account. The Phe $\mathrm{x}$ gender interaction ( $1=$ male, $0=$ female) did not show any relationship between these predictors and lipid profile. Phe $\mathrm{x}$ SD refers to the change in the outcome for a 1SD increase in Phe and showed no significant correlation with four lipid fractions. We found there was a mild positive correlation between BMI and total cholesterol, LDL-cholesterol and triglycerides. There was a negative correlation between BMI and HDL-cholesterol, the finding also observed in a nonPKU population (Table 2).

\section{Discussion}

Our findings support the concept that there was no association between plasma Phe concentration and lipid profile in a large cohort of adult patients with PKU. In this paper we discuss the effect these individual factors had on cholesterol concentrations.

This study was the first to examine the effect of Phe on lipid profile in such a large cohort of adult patients with PKU. Consideration of several confounding factors in the analysis was one of key advantages of the methodology (Table 2). Apart from examining the effect of gender on Phe concentrations, we also assessed the effect of the interaction Phe $\mathrm{x}$ gender on four lipid fractions. The aim was to allow for the possibility that the relationship between Phe might vary according to these variables.

In view of the broad spread of Phe ranges (130-1906 $\mu \mathrm{mol} / \mathrm{L})$, Phe results were presented as $1 \mathrm{SD}$ to express the change in the outcome for a $1 S D$ increase in Phe.

\section{Cardiovascular risk}

We demonstrated that irrespective of plasma Phe concentrations, serum total cholesterol and LDL-cholesterol concentrations were decreased in our patients with PKU. These findings were comparable with recent studies on adult patients with PKU $[19,20]$. Artuch et al. [5] has previously shown that the levels of their lipids, lipoproteins and apolipoproteins indicated a less atherogenic profile. LDL-cholesterol levels were previously shown to be significantly lower in patients with PKU compared to healthy population $[5,15,16]$. Additionally, LDLcholesterol/ApoB ratio was higher in PKU patients who had good dietary compliance when compared to those PKU patients who had a relaxed diet. High LDL-cholesterol/ApoB ratio usually correlates with the presence of larger and less atherogenic LDL particles, which are less susceptible to oxidative damage than small LDL particles [21].

In our study the mean total cholesterol was significantly lower in adult PKU patients when compared to cholesterol targets outlined for normal population. The mean serum LDL-cholesterol was $2.5 \mathrm{mmol} / \mathrm{L}$ and was well within the target of $3 \mathrm{mmol} / \mathrm{L}$ for healthy non-PKU

\begin{tabular}{|c|c|}
\hline Baseline characteristics $(\mathrm{n}=176)$ & Mean \pm SD \\
\hline Total cholesterol & $4.33 \pm 0.94$ \\
\hline LDL-cholesterol & $2.48 \pm 0.8$ \\
\hline LDL-cholesterol in 'PKU diet group' & $2.37 \pm 0.75$ \\
\hline LDL-cholesterol in 'off PKU diet group' & $2.6 \pm 2.8$ \\
\hline HDL-cholesterol & $1.2 \pm 0.34$ \\
\hline Triglycerides & $1.6 \pm 0.96$ \\
\hline Gender (F/M) & $91 \mathrm{~F} / 85 \mathrm{M}$ \\
\hline Age & $32.5 \pm 10.75$ \\
\hline BMI (kg/m $\left.{ }^{2}\right)$ & $28.6 \pm 7.1$ \\
\hline Phe ( \pm SD) & $1017 \pm 440.7$ \\
\hline PKU diet & $82(46 \%)$ \\
\hline Off PKU diet & $94(54 \%)$ \\
\hline
\end{tabular}

Table 1: Baseline characteristics. 
population [13]. Further studies are required to determine whether this effect confers the reduced cardiovascular risk. Nevertheless no evidence of ischemic disease or past medical history of cardiovascular events was observed in our patients and hypertension was not a problem either.

\section{Diet}

The outcomes of our study fully support the findings by Williams et al. [22], who demonstrated that compliance with dietary restriction did not appear to impact on plasma cholesterol concentrations [22]. We showed that our PKU patients, who had good adherence to their diet, were not at high risk of developing atherosclerosis because their mean LDL-cholesterol was low at $2.38 \mathrm{mmol} / \mathrm{L}$. Although the difference was not statistically significant $(\mathrm{p}=0.15)$, the value was lower from the mean LDL-cholesterol $(2.6 \mathrm{mmol} / \mathrm{L})$ found in patients who were not following PKU diet. These results contradict the previously described findings by Artuch et al. [5] and suggested that protein-restricted diet and Phe restriction, associated with a generally healthy lifestyle, may reduce serum LDL-cholesterol [5].

Importantly, the mean triglycerides concentration was 1.6 $\mathrm{mmol} / \mathrm{L}( \pm 0.9)$. Higher triglyceride values in young patients with PKU were reported previously and were believed to be secondary to the significantly high calories consumption from carbohydrates [23]. It should be emphasized that serum cholesterol concentrations depend on a vegetarian diet consisting of olive oil and cream cheese. Notably, the increased total percentage of calories from fat in the form of monounsaturated fatty acids in the diet, and as a result lowering the polysaturated fatty acids and carbohydrates, may have a more beneficial effect on all fractions of lipid profile including serum HDLcholesterol and triglycerides [24]. Lifelong protein restriction may lead to chronic deficit of omega- 3 and omega- 6 fatty acids with the risk of early atherosclerosis. Htun et al. [17], however, have shown that despite low HDL-cholesterol concentration and normal carotid intimal thickness adolescent PKU patients who avoid fish in their diet have low risk atherosclerotic risk [17].

\section{Age and gender}

It was previously demonstrated that with age, patients find it difficult to comply with low-protein diet that may impact on their serum lipid profile [10] but the correlation between serum cholesterol and age, plasma Phe and has never been proven [8]. Our study did not show any considerable association between age and four lipid profiles (Table 2).

Although we did not observe a correlation of any statistical significance between gender and four fractions of the lipid profile, we noted that total cholesterol, LDL-cholesterol and HDL-cholesterol may be increased in male patients by $0.03,0.023$ and $0.007 \mathrm{mmol} / \mathrm{L}$, respectively, levels which are not clinically significant. The Phe $\mathrm{x}$ gender interaction did not show any association between gender and total cholesterol, LDL-cholesterol, HDL-cholesterol and triglycerides (Table 2).

\section{BMI}

Artuch et al. [5] showed that a high percentage of patients with low Phe tolerance and good dietary compliance had raised BMI, the finding not perceived in our study [5]. Couce et al. [25] noticed that biochemical parameters such as total cholesterol, triglycerides, LDL-cholesterol were higher in overweight or obese patients with PKU [2]. We documented a positive correlation between BMI and total cholesterol, LDL-cholesterol and triglycerides and a negative correlation between BMI and HDL-cholesterol. Among our patients with PKU, the range of BMI ranged from 18.9 to $46.9 \mathrm{~kg} / \mathrm{m}^{2}$. A variety of other lifestyle modifying (lack of exercise and alcohol consumption) and genetic factors could be a potential explanation of our observations.

One of limitations of this study is the lack of evidence of possible genetic influence in cholesterol metabolism or underlying liver disease. They should be considered as important variables in interpreting lipids data from PKU patients [24]. Additionally, as we did not have any control group, we compared results to targets for total cholesterol and LDL-cholesterol for healthy population as outlined in guidelines.

In conclusion, the outcomes of the study demonstrate that adult PKU patients have low serum cholesterol concentration and low incidence of cardiovascular events irrespective of diet or their BMI. Lipid profile in adult PKU patients should be requested only if there is

\begin{tabular}{|c|c|c|c|c|}
\hline & $\begin{array}{l}\text { Total Cholesterol } \\
\text { Coefficient }(95 \% \mathrm{Cl})\end{array}$ & $\begin{array}{c}\text { HDL Cholesterol } \\
\text { Coefficient }(95 \% \mathrm{CI})\end{array}$ & $\begin{array}{l}\text { LDL Cholesterol } \\
\text { Coefficient }(95 \% \mathrm{CI})\end{array}$ & $\begin{array}{c}\text { Triglycerides } \\
\text { Coefficient }(95 \% \mathrm{Cl})\end{array}$ \\
\hline Intercept & 2.517 (1.771 to 3.263 ) & $1.311(1.026$ to 1.595$)$ & $0.905(0.189$ to 1.622$)$ & $0.68(-0.212$ to 1.573$)$ \\
\hline BMI & $0.028(0.009$ to 0.047$)$ & $-0.007(-0.014$ to 0$)$ & $0.017(-0.001$ to 0.035$)$ & $0.032(0.011$ to 0.054$)$ \\
\hline Phe & $0(0$ to 0$)$ & $0(0$ to 0$)$ & $0(0$ to 0.001$)$ & $0(-0.001$ to 0$)$ \\
\hline Phe $\times$ SD* & $0.003(-0.179$ to 0.185$)$ & $-0.019(-0.088$ to 0.051$)$ & $0.139(-0.033$ to 0.312$)$ & $-0.129(-0.346$ to 0.088$)$ \\
\hline Gender & 0.03 (0.018 to 0.042$)$ & 0.007 (0.003 to 0.012$)$ & $0.023(0.012$ to 0.035$)$ & $0.002(-0.012$ to 0.017$)$ \\
\hline Age & $0.224(-0.503$ to 0.951$)$ & $-0.113(-0.39$ to 0.165$)$ & $0.453(-0.225$ to 1.131$)$ & $0.228(-0.613$ to 1.068$)$ \\
\hline Gender $\times$ Phe & $0(-0.001$ to 0.001$)$ & $0(0$ to 0$)$ & $0(-0.001$ to 0$)$ & $0(-0.001$ to 0.001$)$ \\
\hline
\end{tabular}

"Phenylalanine results refer to the change in the outcome for a 1 SD increase in Phenylalanine

Table 2: The relationship between predictors and four outcomes (total cholesterol, LDL-cholesterol, HDL-cholesterol and triglycerides). Results were expressed as coefficient $( \pm 95 \%$ Confidence Interval). The analysis was performed using multiple linear regression. 
a strong suspicion of cardiovascular disease or a strong family history of lipid disorder. No observed correlation between Phe concentration, adherence to low-protein diet, BMI, age, gender and lipid profile in our cohort of adult patients with PKU, indicates that there are other factors affecting lipid profile in male and female patients with PKU. Further research is needed to investigate mechanisms of hypocholesterolaemia in this cohort of patients.

\section{References}

1. Van Spronsen FJ, van Wegberg AMJ, Ahring K, Belanger-Quintana A, Bosch $A M$, et al. (2017) Key European guidelines for the diagnosis and management of patients with phenyloketonuria. Lancet Diab Endocrinol 16: 30320-30325.

2. Galluzzo CR, Ortisi MT, Castelli L, Agostoni C, Longhi R (1985) Plasma lipid concentrations in 42 treated phenylketonuric children. $\mathrm{J}$ Inherit Metab Dis 8 : 129 .

3. Acosta PB, Alfin-Slater RB, Koch R (1973) Serum lipids in children with phenyloketonuria (PKU). J Am Diet Assoc 63: 631-635.

4. Castillo M, Zafra MF, Garcia-Peregrin E (1988) Inhibition of brain and liver 3-hydroxy-3-methylglutaryl-CoA reductase and mevalonate-5-pyrophosphate decarboxylase in experimental hyperphenylaninaemia. Neurochem Res 13 $551-555$

5. Artuch R, Colome C, Sierra C, Brandi N, Lambruschini N, et al. (2004) A longitudinal study of antioxidant status in phenylketonuric patients'. Clin Biochem 37: 198

6. Shefer S, Tint GS, Jean-Guilliaume D, Daikhin E, Kendler A, et al. (2000) Is there a relationship between 3-hydroxy-3-methylglutaryl coenzyme A reductase activity and forebrain pathology in the PKU mouse? J Neurosci Res 61: 549563.

7. Hargreaves IP (2007) Coenzyme Q10 in phenyloketonuria and mevalonic aciduria. Mitochondria 78: S175-S180.

8. Colome C, Artuch R, Lambruschini N, Cambra FJ, Campistol J, et al. (2001) Is there a relationship between plasma phenyloalanine and cholesterol in phenyloketonuric patients under dietary treatment? Clin Biochem 34: 373-376.

9. DeClue TJ, Davis J, Schocken DM, Kangas R, Benford SA, et al. (1991) Serum lipid concentrations in subjects with phenyloketonuria and their families. AM J Dis Child 145: 1266-1268.

10. Giovannini M, Agostini C, Bellu R, Ghiglioni R, Giuffre D, et al. (1993) Nutritional aspects of phenylketonuria: correlation between blood lipids and immunoglobulins $G, A$ and $M$ in a phenylketonuric paediatric population on diet therapy. Dev Brain Dysfunction 6: 168-171.

11. Schulpis $\mathrm{KH}$, Karakonstantakis T, Bartzeliotou A, Karikas GA, Papassotiriou I (2004) The association of serum lipids, lipoproteins and apolipoproteins with selected trace elements and minerals in phenylketonuric patients on diet. Clin Nutr 23: 401-407.
12. Verduci E, Agostoni C, Biondi ML, Radaelli G, Giovannini M, et al. (2004) Apolipoprotein $B$ gene polymorphism and plasma lipid levels in phenylketonuric children. Prostaglandins Leukot Essent Fatty Acids 71: 117-120.

13. Perk J, De Backer G, Gohlke H, Graham I, Reiner Z, et al. (2012) European Guidelines on cardiovascular disease prevention in clinical practice. Eur Heart J 33: 1635-1701.

14. Kanufre VC, Soares RDL, Alves MRA, Aguiar MJB, Starling ALP, Norton RC (2015) Metabolic syndrome in children and adolescents with phenylketonuria. J de Pediatria 91: 98-103.

15. Verdici E, Banderali G, Moretti F, Lassandro C, Cefalo G, Radaelli G, et al. (2016) Diet in children with phenylketonuria and risk of cardiovascular disease: a narrative overview. Nutr Metab Cardiovasc Dis 26: 171-177.

16. Gunduz M, Aakar S, Kuyum P, Makav B, Arslan N (2016) Comparison of atherogenic risk factors among poorly controlled and well-controlled adolescent phenylketonuria patients. Cardiol Young 26: 901-908.

17. Htun P, Nee J, Ploeckinger U, Eder K, Geisler T, Gawaz M, et al. (2015) Fish-free diet in patients with phenylketonuria is not associated with early atherosclerotic changes and enhanced platelet activation. PLoS One 10: e0135930.

18. Steyerberg EW, Harrell FE, Borsboom GJ, Eijkemans MJC, Vergouwe Y, et al (2001) Internal Validation of Predictive Models: Efficiency of some procedures for logistic regression analysis. J Clin Epidem 54: 774-781.

19. Nagasaka H, Tsukahara $H$, Taktani $T$, Sanayama $Y$, Takayanagi $M$, et al. (2011) Cross- sectional study of bone metabolism with nutrition in adult classical phenylketonuric patients diagnosed by neonatal screening. J Bone Miner Metab 29: 737-743.

20. Nagasaka H, Okano $Y$, Kimura A, Mizuochi T, Sanayama Y, et al. (2013) Oxysterol changes along with cholesterol and vitamin $D$ changes in adult phenylketonuric patients diagnoses by newborn-mass screening. Clin Chem Acta 416: 54-59.

21. Campos H, Genest J, Blijlevens E, McNamara JR, Jenner JL, et al. (1992) Low density lipoprotein size and coronary artery disease. Arterioscler Thromb 12 : 187-195.

22. Williams RA, Hooper AJ, Bell DA, Mamotte CD, Burnett JR (2015) Plasma cholesterol in adults with phenyloketonuria. Pathology 47: 134-137.

23. Schulpis KH, Scarpalezou A (1989) Triglycerides, cholesterol, HDL, LDL, and VLDL cholesterol in serum of phenyloketonuric children under dietary control. Clin Pediatr (Phila) 28: 466-469.

24. DeClue TJ, Davis J, Schocken DM, Kangas R, Benford SA (1992) Serum lipid concentrations in subjects with phenyloketonuria and their families. AM J Dis Child 145: 1266-1268.

25. Couce ML, Vitoria I, Aldámiz-Echevarría L, Fernandez-Marmiesse A, Roca I, et al. (2016) Lipid profile status and other related factors in patients with Hyperphenylalaninaemia. Orphan J Rare Dis 11: 123. 